\title{
Survey on TOEFL android apps for higher education
}

\author{
Alimin and Irfan Syamsuddin* \\ School of Electrical Engineering, State Polytechnic of Ujung Pandang, Makassar, Indonesia
}

(C2017 ACCENTS

\begin{abstract}
Currently mobile applications have achieved high popularity in various aspects such as education. The use of androidbased applications in the world of higher education is argued to be relevant to improve the quality of teaching and learning processes. This is a preliminary study to evaluate the long-term impacts of the use of android-based applications in teaching English especially in vocational higher education. In particular, this study presents a simple survey of several TOEFL android apps in terms of their features, advantages and disadvantages. It is expected that the survey will be a guide for further research developments in this field.
\end{abstract}

\section{Keywords}

TOEFL, Android application, Vocational higher education.

\section{Introduction}

In the last two decades the use of mobile devices has increased dramatically. The wide range of features that mobile devices offer are based on the so called convergence technology which enables a variety of task that previously require multiple and separate tools can be done with a single device only [1].

Today's mobile devices that adopt cutting-edge technology can be seen everywhere used by every age in every place. Attractiveness of mobile devices enhanced also by strict competitions among the producers which in turn affects the price to be more affordable to lower income people. The combination of high technologies and affordability are perfect to make mobile devices commonly used in doing communications, extending business, managing schedule, monitoring health, and education particularly in higher education.

Internet access through smartphones produces an unlimited number of learning resources but has not received serious attention in the form of a structured and continuous research and involves experts from different areas of expertise.

This fact attracts researchers and educators to make continuous study on the impact of smartphone in learning as well as suggesting how the technology may lead to better results in education.

*Author for correspondence

155
Teaching English is one among many fields that has been greatly helped by the advancement of smartphone technologies [2].

This study is a preliminary effort of future multidisciplinary research that focuses on evaluating the extent to which the android apps for TOEFL are available both free and paid will have a positive impact on developing English proficiency for students in vocational colleges. The main aim of this preliminary study is to survey a number of TOEFL Android apps that potentially improving students' language skills particularly in vocational higher education.

The rest of this paper is structured as follows. Section 2 presents theoretical background of digital learning. Then, in section 3 TOEFL as world English standard skill is explained, followed by survey of a number of current TOEFL Android Apps in section 4. Finally, conclusion is given in the last section.

\section{Digital learning era}

The introduction of the Internet has brought revolution in information industry. Internet has affected many areas, and education is no exception. Internet has become tool and media at the same time for digital learning style. Internet as media is the container of a message that the source or its intended distributor wants to forward to the target or recipient of the message, the material to be conveyed is a learning message transformed in digital formats, 
while the goal to be achieved is the occurrence of the learning process [3].

Learning media in general is a tool of teaching and learning process. Everything that can be used to stimulate the mind, feelings, attention and ability or skills of learners so as to encourage the learning process. Media is the container of a message that the source or its intended distributor wants to forward to the target or recipient of the message, the material to be conveyed is a learning message, and that the goal to be achieved is the occurrence of the learning process. It can be concluded that the learning media is anything that can channel the message, can stimulate the mind, feelings, and willingness of learners so as to encourage the creation of learning process in the learners themselves.

\subsection{Type of digital learning media}

There are at least three types of digital learning media that mainly applied in today's academic environment as follows [4]:

\section{a. Audio media}

Audio media is a medium that the content of the message is only received through the sense of hearing. Judging from the nature of received messages, audio media can convey verbal messages (spoken or verbal language) or non-verbal (sounds and vocalizations).

\section{b. Visual media}

Visual media is a media that only relies on the sense of sight. Visual media looks its material using projection tool or projector, because through this media software (software) which equip this projection tool will produce a bias of light or picture in accordance with the desired material.

\section{c. Audio-visual media}

Audio-visual media is also used as a video media. Video is a medium used to convey learning messages. In the video media there are two elements of mutual unity that is audio and visual. The presence of audio elements allows students to be able to receive learning messages through hearing, while the visual element allows the creation of learning messages through the form of visualization.

\subsection{Technologies for digital learning media}

Mobile technologies offer many benefits as a digital learning media. This is due to advanced functions and features which are well integrated into a smartphone this time. With such facilities and features, the mobile technologies have strong chance to be used as core technology in current learning environment. In short, smartphone offers the following benefits for learning tools include [4]:

- As a counting tool (replacing the calculator).

- As a tool to translate the language digitally.

- As a medium of communication and a means of guidance of students (SMS remind students to do homework, for time to learn, etc.)

- As a tool to take pictures / photos for learning materials.

- Thanks to advance processor technology, particular smartphone even equipped with high speed processor which previously only seen in PC. Mobile phones become one alternative for PC.

- Teachers can create blogs (web logs, notes on internet sites) via mobile phones.

- Students can search for information on the internet via mobile phone.

- Timetabling and reminder functions are important for class schedule.

- Data storage can be done with mobile phone memory or even via cloud computing features.

\section{TOEFL}

TOEFL stands for test of English as a foreign language, is one of the test models English used for measure their level of skill who does not use English as the mother tongue (non-native speakers) [5].

The Test of English as a Foreign Language, or TOEFL, is a test which measures people's English language skills to see if they are good enough to take a course at university or graduate school in Englishspeaking countries. It is for people whose native language is not English. It measures how well a person uses listening, reading, speaking and writing skills to perform academic tasks. This test is accepted by more than 7,500 colleges, universities, and agencies in more than 130 countries; which mean it is the most widely recognized English test in the world [5].

The format of the TOEFL test has been changed three times. The first was the PBT (paper-based TOEFL test). It tests listening, reading and grammar skills with a perfect score being 677. Some centers where computers are not available still offer this format [5]. The second format is the CBT (computer-based TOEFL test). People are each provided with a computer to take the test. A writing section was added as well as the three sections. The level of listening and grammar skills are automatically changed depending on a person's English level. The third change is the iBT (Internet-based test) that is being brought in around the world which measures 
listening, speaking, reading and writing[5][6]. The TOEFL score conversion table to know the TOEFL score is summarized in Table 1 [6].

Table 1 Summary of TOEFL score

\begin{tabular}{lll}
\hline Skill & Score range & Level \\
\hline Reading & $0-30$ & High(22-30) \\
& & Intermediate(15-21) \\
& & Low(0-14) \\
\hline Listening & $0-30$ & High(22-30) \\
& & Intermediate(15-21) \\
& & Low(0-14) \\
\hline Speaking & $0-30$ score scale & Good(26-30) \\
& & Fair(18-25) \\
& & Limited(10-17) \\
& & Weak(0-9) \\
\hline Writing & $0-30$ score scale & Good(24-30) \\
& & Fair(17-23) \\
& & Limited(1-16) \\
\hline Total Score & $\mathbf{0 - 1 2 0}$ & \\
\hline
\end{tabular}

\section{TOEFL android applications}

\subsection{Android}

Android is an open-source software platform which was introduced by Google in 2007. Unlike iOS and Windows, Android's open-source approach contrasts with Apple's curated environment. Open-source software lets Android users to share resources and products and also able to tailor and customise it [7, $8]$.

Currently, Android has been adopted by many mobile device manufacturers such as Samsung, Sony Ericsson, LG, Dell, Huawei, Fujitsu Toshiba, Motorola, Panasonic, Acer, Asus, NEC, Kyocera, and others.

Its third-party applications are written in a customised version of the Java programming language and can be acquired by users either through an app store, such as Google Play or the Amazon Appstore, or by downloading and installing the application's APK file from a third-party site [8]. The Play Store application allows users to browse, download and update apps published by Google and third-party developers, and is pre-installed on devices that comply with Google's compatibility requirements [8].

\subsection{TOEFL android apps}

The survey takes only free applications since these are the most widely downloaded by users without having to pay anything. There are seven related application described in the following sub section.

\subsubsection{Magoosh TOEFL}

Magoosh TOEFL is an android application that provides various lessons for those who want to improve TOEFL score (Figure 1). It covers all four sections of the test (reading, listening, speaking, and writing). Students may track their progress as they study.

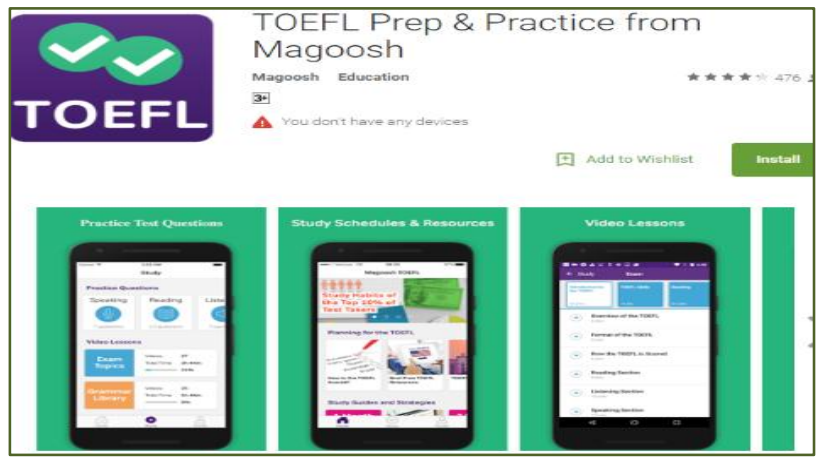

Figure 1 Magoosh TOEFL

To help students understand the section, it also provides more than 100 animated video lessons in approximately 10 hours. The speed of these videos can be adjust for easier listening. In addition there are over 40 lessons in grammar section with detailed review. Detailed explanation of every question type is well given. Finally, student may perform full TOEFL exam, by trying more than 250 TOEFL practice questions available in Magoosh website [9]. 4.2.2 Mobogenie TOEFL speaking master

The next app is by Mobogenie, called TOEFL speaking master [9]. The app is specifically designed to guide students to understand main points of speaking test of TOEFL (Figure 2).

The app is well developed in terms of usability and mobility and it offers three modes for users as follows below.

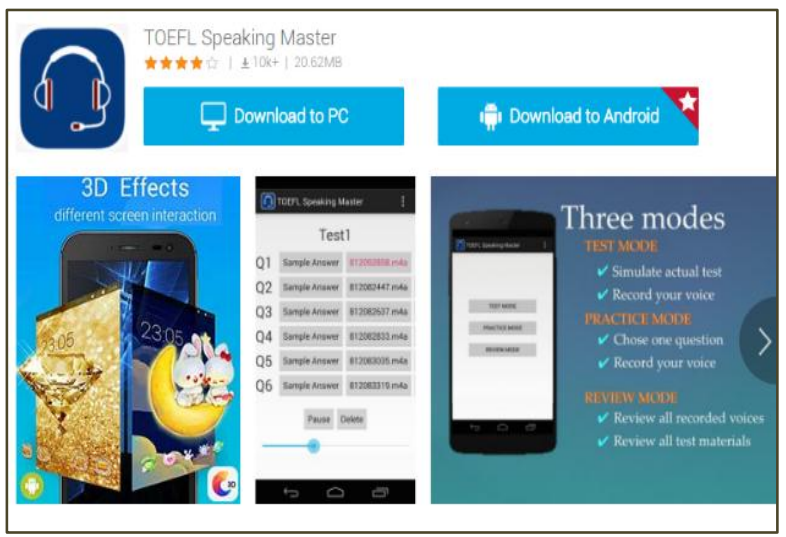

Figure 2 TOEFL speaking master 


\section{a. Test mode}

In this mode, user can perform an actual TOEFL speaking section in simulated environment. User may choose a number of questions and give answer on each which is quite convenient to get to actual TOEFL test. The whole test mode requires maximum 20 minutes to finish.

\section{b. Practice mode}

In practice mode, user may pick a question for practicing. This mode lets user focuses on particular weak point in order to improve it by having adequate practices. Practice mode is ideal for those who do not have lots of time to take whole test of TOFL speaking test.

\section{c. Review mode}

In review mode, user may review all aspects not only voice, but also all study materials such a text, scripts, and sample answers. This mode is very useful to understand all materials without attending test mode or Practice Mode before. Also, by listening your own voices, you can realize your strong points and week points.

Overall, this app is excellent choice for those who want to improve speaking level in spare time.

\subsubsection{EDUBot TOEFL $\mathrm{iBT}$ reading}

The main focus of this app is providing adequate reading exercises for the TOEFL iBT test (Figure 3). It has 34 reading practice tests, all in iBT real format [9].

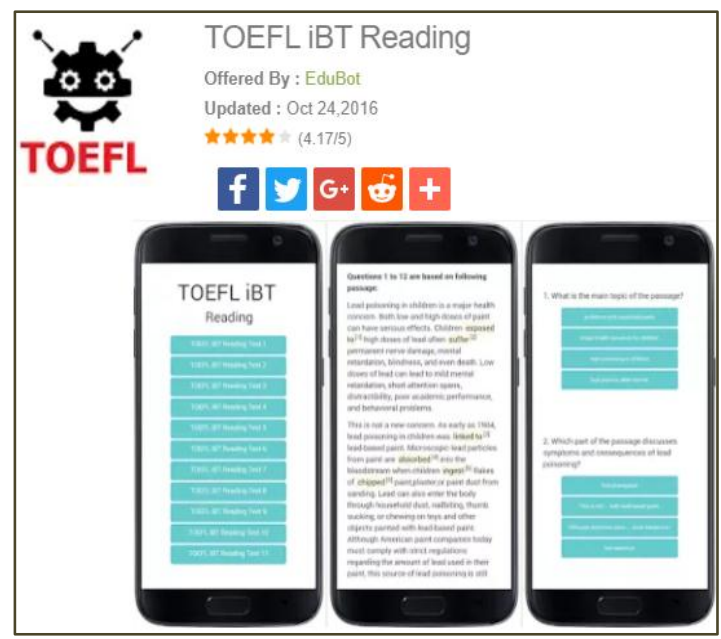

Figure 3 TOEFL iBT reading

In each test, there will be 4 passages to read then choose the correct answers for the questions that follow the passage. In total there are 29 to 30 questions in each test. Commonly, one point will be got if the answer is correct; however, there are particular questions with two points for correct choice.

There is no time limit for the reading practice test but it is suggested complete each test within 40 minutes.

After all, user will gain the total score once finishing the test. In addition, further review also provided for incorrect answer to let user understand weaknesses points. Final feature of this app is recording result for user to evaluate progress.

4.2.4TOEFL grammar test

This app is simply focus in structure base test of TOEFL [9] (Figure 4). It is very easy to use which provides 3 different levels: easy, medium, and hard.

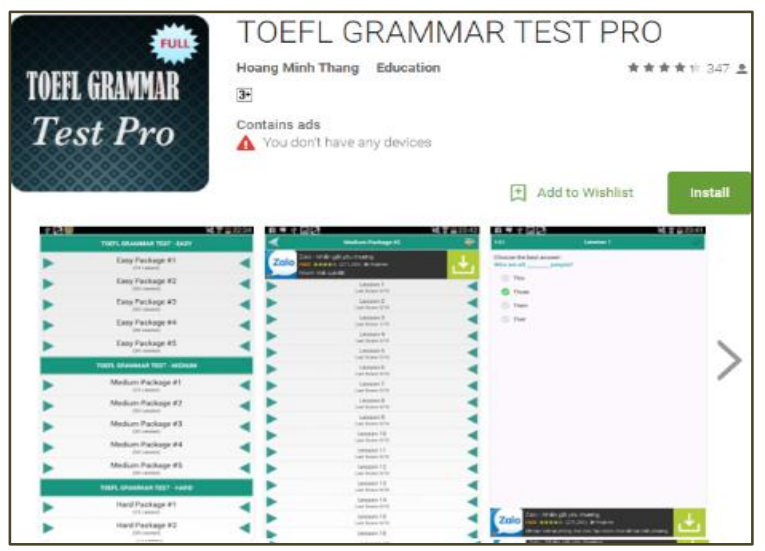

Figure 4 TOEFL grammar test

Similar to other apps, every test in each level has ten questions, while each question has four answers to choose. If the answer is correct, user will gain one point.

Finally, user may obtain the final result which show total score gained. Review of wrong answer also provided in order to learn from mistakes and improve TOEFL structure's abilities in the future.

\subsubsection{TOEFL flash card}

TOEFL Flash Card is an education app aims to help learners understand the TOEFL in a game alike setting [9] (Figure 5). It contains 1600 premade flashcards in two main categories, namely Vocabulary and Reading.

User may play the cards while assessing his or her knowledge through the game card. Beside card, this app also comes with slideshows and quizzes as interactive game to improve vocabulary and reading skills. 
In addition, users can also make their own flash cards, slideshows, take quizzes and much more to learn in an exciting way.

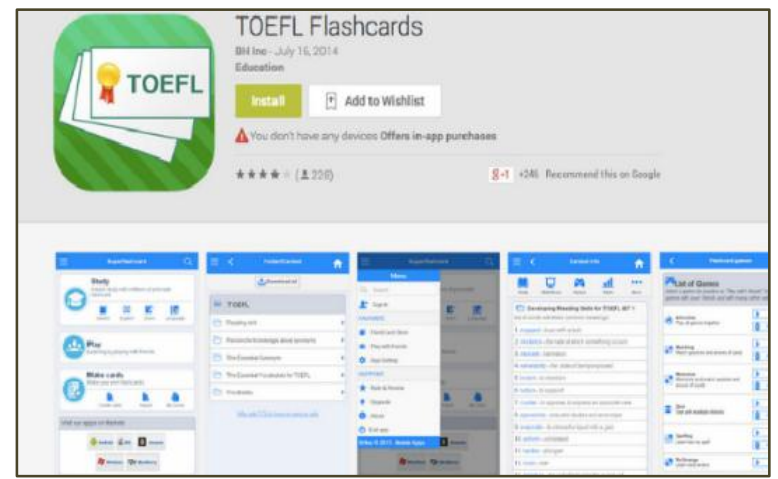

Figure 5 TOEFL flashcards

4.2.6TOEFL incorrect words

TOEFL Incorrect Words is a specific TOEFL app to spot the correct word among many incorrect ones in a sentence or passage [9] (Figure 6).

The app is built simply which makes it easy to use at user side. It contains 1670 questions of incorrect words to practice with. Review also given once user finished the test. Through the review any mistakes can be identified and user can rectify the incorrect words. Overall, this simple app is excellent to help improving TOEFL score.

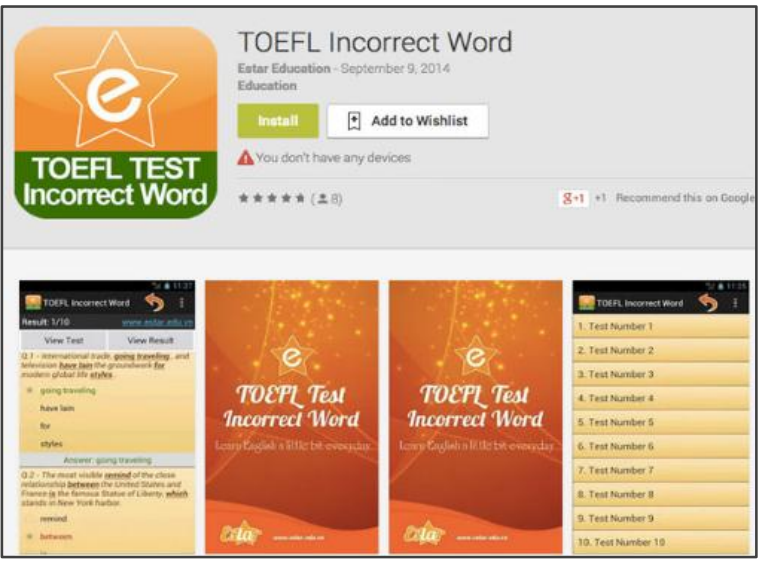

Figure 6 TOEFL incorrect word

\subsubsection{TOEFL pictionary}

TOEFL Pictionary is unique app which functions as a dictionary of the most common TOEFL words with their picture definitions [9] (Figure 7). The app is very useful to improve memory by visualizing it with picture for each word will help user remember new words.
The main advantage is that along with the image, each word is explained in written text with examples as well.

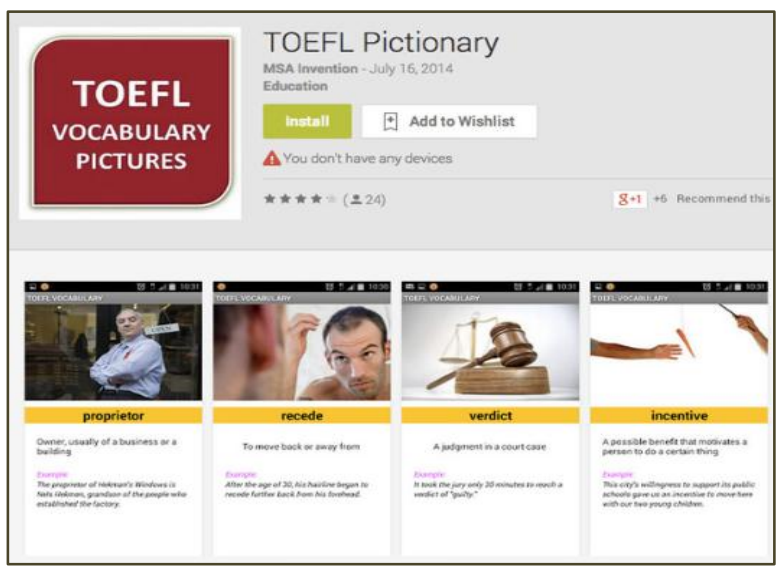

Figure 7 TOEFL pictionary

\section{Conclusion}

The aim of this paper is to present a survey on TOEFL android applications that are available for free and have been downloaded by many users. The survey is intended for describing TOEFL supporting tools for vocational higher education students.

The results found seven apps with various objectives and different features that could help users to improve TOEFL skills such as listening, speaking, and reading but none for writing. While users need to combine them appropriately according to different needs and requirements to improve TOEFL skills and obtain better score. In the future, this study will be extended by having a number students at different age as participants and evaluate their performance after learning from these application for particular period of time.

\section{Acknowledgment}

None.

\section{Conflicts of interest}

The authors have no conflicts of interest to declare.

\section{References}

[1] Hyeongjik LE, Lee WB, Kweon SC. Conjoint analysis for mobile devices for ubiquitous learning in higher education: the Korean case. TOJET: The Turkish Online Journal of Educational Technology. 2013; 12(1):45-51.

[2] Indonesia M. Assessing Moodle as learning management system platform for English course based TOEFL. International Journal of Computer Trends and Technology. 2014; 18(6):276-9. 
Alimin et al.

[3] Syamsuddin I. Fuzzy multi criteria evaluation framework for E-learning software quality. Academic Research International. 2012; 2(1):139-47.

[4] Clark RC, Mayer RE. E-learning and the science of instruction: proven guidelines for consumers and designers of multimedia learning. John Wiley \& Sons; 2016.

[5] Sharpe PJ. Barron's TOEFL iBT: Internet-based test. Barron's educational series; 2010.

[6] Sanchez E. Mastering skills for the TOEFL iBT 2nd edition advanced writing (book review). Accents Asia. 2011:82-4.

[7] Android, Open Source Project. http://source.android.com/about/index.html\#top. Accessed 11 July 2017.

[8] Android, Android Compatibility. http://developer.android.com/guide/practices/compatib ility.html. Accessed 11 July 2017.

[9] Google Play Store. https://play.google.com/. Accessed 13 July 2017

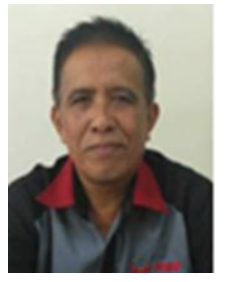

Dr. Alimin, is a senior lecturer School of Electrical Engineering, State Polytechnic of Ujung Pandang PNUP, Indonesia. His research area are English for Academics, Curriculum Design, and Technology Education.

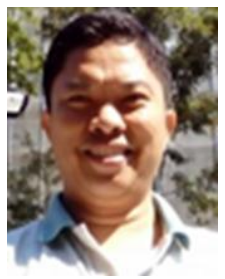

Irfan Syamsuddin, $\mathrm{PhD}$ is a lecturer at School of Electrical Engineering, State Polytechnic of Ujung Pandang PNUP, Indonesia. His research area are Information Technology, Information Security, ELearning, EGovernment and Human Computer Interaction. He is the head of Center for Applied ICT Research (CAIR) of PNUP Indonesia.

Email: irfans@poliupg.ac.id 